\title{
INVESTIGACIÓN/RESEARCH
}

\section{UNA PRESENTACIÓN DE LAS TEORÍAS SISTÉMICAS DE PRIMERA GENERACIÓN, HASTA LA ECOLOGÍA DE LA COMUNICACIÓN}

\author{
Carmen Gaona Pisonero \\ UCAM (Universidad Católica San Antonio de Murcia).
}

\begin{abstract}
Resumen:
A lo largo de este texto desarrollaremos una idea básica desde la que comprender no sólo los procesos de comunicación humana, sino cualquier fenómeno social: la perspectiva sistémica, que equivaldría a tener una forma de pensamiento de interrelación de los elementos en relación a un todo. La ambición de una teoría sistémica, consiste en atender a la globalidad, a las interacciones entre los elementos más que a las causalidades, a lo que tenemos que adjuntar la complejidad de esa globalidad, es decir observar la complejidad de los sistemas como conjuntos dinámicos con relaciones múltiples y cambiantes. Acción esta última que el observador debe de llevar a cabo desde un pensamiento reflexivo.
\end{abstract}

Palabras clave: Teoría General de Sistemas - Comunicación-Sistema - Entorno, Comunicación Humana - Ecología de la Información

\section{INTRODUCCIÓN}

Antes de profundizar de pleno en la perspectiva sistémica, abordaremos las primeras teorías sistémicas. Como primer hito de este recorrido, nos detendremos en la Teoría General de Sistemas (TGS) de Ludwing von Bertalanffy. Seguidamente nos detendremos en algunos conceptos para alcanzar una definición de sistema y aproximarnos a la acción del sistema desde una base biológica, desde la que comprender en un segundo nivel de reflexión, qué es un sistema social. Este concepto lo abordaremos principalmente desde la perspectiva de Talcott Parsons, así como veremos una aplicación de la perspectiva sistémica en algunos estudios de la comunicación de masas; sobre este último aspecto, hemos de recordar tres autores importantísimos: Melvin Defleur, Ithiel de Sola Pool y Abraham A. Moles. Por último haremos algunas incursiones en las actuales perspectivas sistémicas, como es el caso de la "ecología de la información".

\section{ANTECEDENTES Y CARACTERÍSTICAS DEL SISTEMA}

La noción de sistema, o una vaga idea de esta noción, ya está presente en las sentencias de Aristóteles, Anaxágoras, y Heráclito entre otros. Pero sin duda alguna, se da un salto cualitativo con la obra del matemático y biólogo Ludwing von Bertalanffy (1933), quien se planteó si era posible establecer una lógica de funcionamiento general aplicable a todo sistema, es decir establecer un lenguaje común dentro de todo corpus teórico; desde esta perspectiva construye que la Teoría General de Sistemas (TGS). El 
objetivo de la TGS es que se pudiese aplicar a la biología para el estudio de los sistemas vivos, que la TGS fuese también el lenguaje común para entender la física de los sistemas de objetos, la psicología de los sistemas mentales y conceptuales, la sociología y la antropología de los sistemas sociales, la lingüística de los sistemas lingüísticos, etc. Este enfoque tiene lugar en la década de los cuarenta del pasado siglo $X X$, y lo que es significativo de señalar en esta nueva perspectiva, es la sustitución de la idea de objeto por la de sistema: las ciencias estudian sistemas y relaciones de sistemas, no objetos aislados.

El sistema pasa a ser el eje vertebral del pensamiento sistémico, sobre el que gira la (TGS), teoría que viene definida por Ludwing von Bertalanffy como una teoría lógicomatemática que se propone formular y derivar aquellos principios generales aplicables a todos los sistemas.

Como una primera definición de sistema, podemos decir que el sistema es un conjunto de elementos relacionados que componen un todo; estos elementos mantienen entre sí relaciones de interdependencia. Además, las relaciones de interdependencia entre los elementos, y la totalidad resultante, son regidos por reglas susceptibles de ser expresadas en términos lógicos. Pero la totalidad formada por el conjunto de los elementos no es reductible a la suma de esos elementos, puesto que falta un aspecto importantísimo para entender un sistema, su relación con el entorno, que abordaremos en epígrafes siguientes.

A continuación, presentaremos las características básicas de un sistema, desde la perspectiva de la TGS, que serán complementadas con algunas puntualizaciones hechas desde una perspectiva sistémica actual, tomando como referencia especial la obra de Maturana y Varela (1984):

a) Apertura/ clausura: la apertura y clausura del sistema se establece en términos de complementariedad e intercambio, tanto de materia, energía o información. En base a esta característica, hablaremos de los límites del sistema, es decir, su frontera y borde, respecto al entorno.

b) Interacción: En modo alguno la vida de un sistema es posible sin la conexión con otros sistemas. Éstos no se encuentras aislados unos de otros, puesto que además de que en la pluralidad reafirma su identidad, también la reconstruye desde dicha convivencia creativa y de retroalimentación constante.

c) Determinismo estructural: Un sistema no puede cambiar de una forma que no sea posible para su estructura. A su vez, los cambios afectan a la estructura de un sistema, es decir, a los elementos y relaciones que lo componen. Todo lo que ocurre dentro de un sistema ocurre en la forma de cambios estructurales determinados en su estructura, ya sean cambios propios o desarrollados en sus interacciones con el medio, pero no determinados por éste último.

De acuerdo a Maturana, desde una perspectiva biológica, los sistemas vivientes son sistemas determinados estructuralmente. Por lo tanto, todo lo que ocurre en ellos, ocurre en cada instante como parte de su dinámica estructural de ese momento, y está determinado por ese momento. Esto implica que todos los cambios estructurales que un sistema viviente sufre como consecuencia de sus interacciones con su ambiente, no están 
determinados por los agentes externos que el observador ve como actuando sobre él, sino que están determinados por la dinámica estructural del ser vivo (Maturana, 1975).

d) Organización. Según la TGS, un sistema se define por una relación específica entre su estructura y su operación (función) que llamamos organización. En los discursos actuales, en una apertura para integrar la complejidad inherente a todo sistema, hablaríamos de auto-organización, auto-generación.

e) Historia: La historia del sistema es la historia de los cambios de su estructura "procesos de cambio". Histéresis. La existencia del sistema depende de una complementariedad entre cambio y estabilidad. Puesto que la historia de un sistema se refiere a los cambios de estructura, esta característica está en estrecha relación con el determinismo estructural, tal y como puntualiza Maturana y Varela (1984): "todo sistema existe sólo en la conservación de su adaptación y los cambios del ser vivo, y su interacción al medio (y con otros seres vivos o sistemas) se le llama conservación de la congruencia ya que la historia del ser vivo es la historia de las congruencias con el medio".

f) Acoplamiento estructural: (es un tipo de "interacción). Se da entre los elementos de un sistema y el entorno. Posteriormente a la TGS, se amplía el sentido del acoplamiento estructural, así por ejemplo Luhmann (1996) lo considera como una medida de la interprenetración entre dos subsistemas; en ambos casos forma parte de la auto-organización del sistema.

\section{SISTEMA Y ENTORNO}

Cualquier análisis teórico-sistémico debe consistir en la diferencia entre sistema y entorno, si bien en la actualidad se discreparía sobre una diferencia clara entre ambos. Los sistemas están estructuralmente orientados al entorno, que es donde cohabitan y sin él, no podrían existir, por lo tanto, no se trata de un contacto ocasional ni tampoco de una mera adaptación, o acoplamiento estructural tal y como veremos más adelante.

Hay que resalar la diferencia entre sistema y entorno mediante una segunda diferencia constitutiva: la que existe entre elemento y relación. La existencia, reconocimiento e integración de las diferencias, es una dinámica constante de todo sistema. De la diferencia existente entre sistema y entorno, se pasa a otro plano, para centrarse en la diferencia en un sistema. Este segundo tipo de diferencia, es posible por la pluralidad de acciones que se producen en el interior de un sistema, tales como la autoorganización, la auto-reflexión, la auto-redefinición y la auto-catálisis. A continuación ofreceremos unas brevísimas definiciones sobre estos conceptos:

Auto-organización: (desde la perspectiva de José Amozurrutia): Función o acción interna de un "sistema", por la que éste establece, introduce, crea y mantiene el sentido y la coherencia de su función dentro del entorno donde cohabita. Esta acción principal que acabamos de mencionar, desde una óptica comunicativa sería la auto-reflexión, que diseña y mantiene el pulso de la red simbólica del sistema. 
A su vez en la "auto-organización", el sistema establece y mantiene una relación con los elementos del entorno, es decir, la red de acoplamiento con el entorno ${ }^{1}$. Y por otro lado, la auto-organización de un "sistema" implica también, organizar y mantener una relación con los elementos que organizan el sistema mismo, es decir, la red de pulsos simbólicos. Ésta está destinada a propiciar una reflexividad y a generar informaciones y comunicaciones que son transmitidas en formas de decisiones al entorno, dentro de una subred del acoplamiento estructural del sistema.

Podríamos establecer la siguiente relación:

AUTO-ORGANIZACIÓN= AUTO-REFLEXIÓN+AUTO-CATÁLISIS+ AUTOREFEFINICIÓN

En otro orden, evidenciamos las siguientes correspondencias entre acciones del sistema con formas concretas de éste: la red simbólica, la red de pulsos simbólicos y la red de acoplamiento con el entorno, conceptos que se definirán en el siguiente apartado.

- AUTO-REFLEXIÓN: Relacionada con la RED SIMBÓLICA

- AUTO-CATÁLISIS: Relacionada con la RED DE PULSOS SIMBÓLICOS

- AUTO-REDEFINICIÓN: Relacionada con la RED DE ACOPLAMIENTO CON EL ENTORNO

\subsection{LA NOCIÓN DE SISTEMA}

Una definición general de sistema sería la de un "conjunto de componentes, relacionados entre sí, que tienen un propósito previamente establecido". A dicha definición, le añadiremos tres aspectos importantísimos a la hora de analizar, y reconstruir un sistema:

1. la naturaleza de los componentes,

2. el tipo de relaciones y

3. la naturaleza de su propósito.

Estos tres aspectos son importantísimos tanto en las concepciones sistémicas de organismos celulares como de la sociedad. Ambos pueden ser vistos como sistemas, además entre ellos hay una cadena de "sistemas/subsistemas" que están vinculados en diferentes grados de acoplamiento y constituyen un gran sistema.

\section{DELIMITACION CONCEPTUAL DEL SISTEMA SOCIAL DESDE LA DIVERSIDAD REFLEXIVA}

\footnotetext{
${ }^{1}$ Luhmann, interpreta el "acoplamiento estructural", como una medida de la interprenetración entre dos subsistemas, pero igualmente lo incluye dentro de la auto-organización del sistema.
} 
Un referente en esta temática en sin duda alguna Talcott Parons, pero este sociólogo norteamericano no fue el primer autor en abordar teóricamente un "sistema social", sino que nos tendríamos que remitir al sociólogo y economista italiano Vilfredo Pareto, para llegar luego después a Auguste Comte, Durkheim y Weber, Herbert Spencer.

Desde nuestro punto de vista Talcott Parsons junto a Niklas Luckmann, son los autores que más lejos han ido en la elaboración de una teoría general de sistemas en el campo de las ciencias sociales. El pensamiento de Parsons, se traduce en una teoría compleja, por lo que no es fácil interpretar la obra de autor, esto se debe sobre todo al carácter sumamente abstracto del pensamiento de Parsons, y también a su forma de expresarse por escrito, con revisiones constantes, puesto que sus propuestas evolucionaron sin cesar y en direcciones múltiples y a veces sorprendentes.

Desde la óptica de Talcott Parsons, un "sistema social -reducido a los términos más simples- consiste, pues, en una pluralidad de actores individuales que interactúan entre sí en una situación que tienen, al menos, un aspecto físico o de medio ambiente, actores motivados por una tendencia a obtener, un óptimo de gratificación y cuyas relaciones con sus situaciones -incluyendo a los demás actores- están mediadas y definidas por un sistema de símbolos culturalmente estructurados y compartidos". En esta definición faltaría un aspecto importantísimo de la teoría sistémica de Talcott Parsons, la orientación motivacional, y la orientación de valor que rige el sistema social (en especial el sistema cultural). Parsons concede un papel prioritario a los valores, en una estructura precisa de la acción humana.

Otro autor imprescindible en digamos una teoría social sistémica, es Niklas Luhmann, autor de una gran complejidad, imposible de sintetizar en cuatro ideas -atendiendo al objetivo pedagógico de estas páginas-, pero nos parecería un olvido imperdonable obviar a este autor en este tema. Únicamente enunciar su sistema social luhmanniano que está conformado sólo por comunicaciones que se auto-generan, se auto-organizan y conforman subsistemas sociales autopoiéticos. Invitamos al alumno a la lectura de las obras de Luhmann, todo un desafío intelectual.

\subsection{RECORRIDO HISTÓRICO POR LAS TEORÍAS SISTÉMICAS DE PRIMERA GENERACIÓN, HASTA LA ECOLOGÍA DE LA COMUNICACIÓN (A. MOLES)}

Tal y como ya hemos mencionado, el origen de la concepción sistémica está implícita desde la filosofía aristotélica, pasando por Herbert Spencer (1820-1903). Siendo el biólogo Ludwig Von Bertalanffy, una figura importante al proponer en el 1933, una Teoría General de Sistemas, desarrollándose luego después la propuesta de Bertalanffy en el campo de las ciencias sociales, como es el caso de Talcott Parsons, o de Niklas Luhmann (1927-1998), quien propone una perspectiva sistémica de la sociedad.

En 1933, en una obra titulada Modern Theories of Development, el biólogo Ludwing von Bertalanffy establecía las bases de lo que formalizaría en la posguerra como la "teoría de los sistemas", una teoría cuyos principios han proporcionado un instrumento de acción con fines estratégicos durante la Segunda Guerra Mundial. Bertalanffy usa el término "función", relacionándolo con los "procesos vitales u orgánicos en la medida en 
que contribuyen al mantenimiento del organismo". El sistemismo y el funcionalismo comparten por tanto un mismo concepto fundamental: el de función, que denota la primacía del todo sobre las partes.

Años más tarde, a partir de los años sesenta, investigadores conocidos más directamente como teóricos de la comunicación de masas y de la opinión publica, descubren entonces las virtudes del modelo sistémico y lo aplican en sus estudios sobre el proceso de formación de las decisiones políticas (Lasswell, Bauer Pool y Dexter). En el horizonte de estas preocupaciones surge una reflexión operativa situada en el contexto de la guerra fría; el equilibrio del poder, la seguridad colectiva, el gobierno mundial. La presión de la peritación es tan fuerte que Ithiel de Sola Pool, profesor en el Instituto Tecnológico de Massachusetts MIT, no duda en dedicarse plenamente, a petición del Pentágono, a la formulación de un modelo (Agile-Coin) que alimente las estrategias contrainsurreccionales (Coin es la contracción de Counterinsurgency) en el sudeste de Asia y en América Latina.

El modelo sistémico tiene otras consecuencias menos determinadas para el contexto político internacional. En esos mismos años sesenta, por ejemplo, permite al norteamericano Melvin de Fleur hacer más complejo el esquema lineal de Shannon resaltando la función desempeñada por la "retroalimentación" (feedback) en el "sistema social" que los medios de comunicación de masas en su conjunto constituyen. "Cada uno de los medios de comunicación es en sí mismo un sistema social independiente , pero todos están vinculados entre sí de forma sistemática" (De Fleur, 1966). Continuando con la propuesta de Melvin de Fleur, cada uno de estos conjuntos se representa con sus dos "subsistemas", encargados respectivamente de la "producción" y la "distribución", cada uno de los cuales implica a diversos actores con sus distintos "sistemas de funciones". Entre estos actores, destacan sobre todo las agencias de publicidad, las sociedades de estudios de mercado y de medición de audiencias, y los organismos de regulación y de arbitraje. La preservación del "equilibrio del sistema" condiciona los contenidos.

Veamos en las siguientes líneas más datos sobre tres autores importantísimos en el campo de la Teoría de la Información: Melvin De Fleur, Ithiel de Sola Pool y Abraham

A. Moles.

\section{Melvin De Fleur:}

En 1976, DeFleur, junto con Sandra Ball-Rokeach, describió la teoría de la 'dependencia de los medios', que se argumenta en la estrecha vinculación entre los medios, la audiencia y la sociedad, generadora de una relación de dependencia. Theories of Mass Communication, que escribió con Ball-Rokeach, le consagró como uno de los investigadores pioneros en teoría de la comunicación de masas; tuvo continuidad en Understanding Mass Communication (con Everette Dennis) y Milestones in Mass Communication Research, el texto con más repercusión en su carrera, elegido en 1999, en una encuesta de la AEJMC (Association for Education in Journalism and Mass Communication), como uno de los diez libros más influyentes del siglo $\mathrm{XX}$ en materia de comunicación. 
Abunda en la teoría de los efectos desde el plano de la relación de dependencia existente entre medios y audiencia. El libro profundiza en el origen humano de la comunicación y las transformaciones que apuntan hacia un uso más amplio de los medios, puesto que su planteamiento al establecer las diferentes interconexiones entre cultura, sociedad, política, individuo y medios de comunicación, se establece como un planteamiento sistémico en un sentido simple de perspectiva sistémica. Las repercusiones de los medios no sólo se circunscriben a efectos ideológicos, sino que se proyectan en los valores de consumo y comportamiento, en tanto pueden activar o inhibir las conductas de la audiencia. Los aspectos cognitivos, afectivos y de comportamiento están directamente influidos por la acción mediática. Los efectos apuntan a la expansión de un sistema de creencias mayoritario y la formación de actitudes a través, entre otras vías, de la selección temática y de la agenda de los medios.

Melvin Defleur, recoge aportes de dos autores : Eliu Katz y Paul Lazarsfeld-. De Katz señala la interacción que existe entre las psicologías individuales y las normas sociales; de Lazarsfeld sería su planteamiento teórico de que los medios de comunicación establecen unas normas y que éstas están conectadas a su vez con opciones políticas concretas, con intereses comerciales, etc. Pero el proceso no es tan sencillo, por lo que pone en cuestión la relación estímulo-respuesta del modelo de la 'aguja hipodérmica' en la acción comunicativa como una regla universal y de efectos inmediatos, sentando las bases de la revisión crítica de las futuras teorías sobre la recepción. En DeFleur, la nueva variable decodificadora está en la dimensión psicología del individuo o del grupo, por lo que el éxito de la persuasión comunicativa pasa por trabajar sobre esa variable. Sin embargo, no abandona el esquema causal de sus predecesores, aunque la hace más complejo. De una forma visual, en el siguiente esquema quedaría reflejada su "perspectiva sistémica" sobre medio, audiencia y sociedad:

\section{Ithiel de Sola Pool:}

En la primera mitad de los años setenta, Ithiel de Sola Pool hace progresar la teoría de los sistemas aplicándola al análisis de nuevos planteamientos de organización de la vida política, posibilitados por el desarrollo de la tecnología de la televisión por cable.

Este autor, durante la Segunda Guerra Mundial trabajó con Harold Lasswell en proyectos de investigación sobre las propagandas de los regímenes nazi y soviético. Algunos de sus primeros artículos científicos aparecen firmados junto a los padres de investigación en comunicación (Lasswell, Lazarsfeld y Schramm). Inició su actividad académica en la Universidad de Stanford, pero muy pronto pasó al Instituto Tecnológico de Massachusetts (MIT), donde desarrolló sus más conocidos trabajos en el campo de los efectos de las tecnologías de la comunicación en los nuevos escenarios globales. Creó y presidió el Departamento de Ciencia Política del MIT y el MIT Communications Forum.

Su papel ha sido relevante en el desarrollo de las ciencias sociales, con especial aplicación en el desarrollo de la teoría de redes. Trabajó asimismo en el campo del análisis cuantitativo de los flujos informativos y en la creación de simuladores computerizados para la toma de decisiones en situaciones de crisis. Son también muy notables sus aportaciones acerca de los nuevos usos tecnológicos en los campos de la 
información y la comunicación donde su análisis prospectivo anticipa los riesgos y las oportunidades de la impregnación tecnológica de los usos sociales y culturales, consecuencia del desarrollo de la informática y las telecomunicaciones espaciales. También estudia los cambios en el escenario de las naciones y de las relaciones de poder internacionales. Sola Pool hace referencia a las 'tecnologías de la libertad' y se acerca a los análisis de la economía política de la comunicación, a la naturaleza de los sistemas de medios y al papel creciente que en la economía va adquiriendo la información y las redes de comunicación.

\section{Abraham A. Moles:}

En Francia, Abraham Moles (1920-1992), ingeniero y matemático, sitúa su proyecto de "ecología de la comunicación" a la vez bajo el signo de la teoría matemática de Shannon y de los análisis de Norbert Wiener. La comunicación se define como la acción de hacer participar a un organismo o a un sistema situado en un punto dado $\mathrm{R}$ en las experiencias y estímulos del entorno de otro individuo o sistema situado en otro lugar y otro tiempo, utilizando los elementos de conocimiento que tienen en común.

Antes de llegar teóricamente a esa ecología de la comunicación, Abraham A. Moles, recorre un largo recorrido teórico que se inició con sus trabajos sobre física del Ruido, sobre la Música Experimental, para dar el salto a la Teoría de la Información, en concreto mediante una Teoría informacional de la Percepción, y sobre la Sociodinámica de la Cultura.

El pensamiento de Moles, evoluciona dentro de la línea de Teoría de la Información y Psicología del espacio, hasta llegar a una ecología de la comunicación. En pocas palabras, en esta última propuesta se integra el estudio de la interacción entre especies diferentes en un ámbito dado. Las especies de comunicación, próxima o lejana, fugaz o registrada, táctil o auditiva, personal o anónima, son especies que reaccionan efectivamente entre sí en el espacio cerrado de las veinticuatro horas de la cotidianeidad o el espacio social del planeta.

Esta ecología debería abarcar dos ramas diferentes: La primera tiene como unidad el ser individual y se ocupa de la interacción de las modalidades de su comunicación en su esfera tiempo, la de su balance-tiempo, y su esfera espacio, la de los trayectos en un territorio. La segunda rama se refiere a la organización de la logosfera, grafosfera y videosfera, al condicionamiento del planeta por múltiples canales que ponen los mensajes en circulación y a la sedimentación de estos últimos en los lugares mnemónicos, como archivos o bibliotecas.

Tras la presentación de estos tres autores, hemos finalizado este veloz recorrido por las implicaciones sistémicas en el campo de la teoría de la comunicación, que finaliza en la "ecología de la comunicación. Cabe evidenciar que la brevedad no debe ensombrecer la relevancia que esta teoría supone, así como su repercusión e influencia actual en el campo de las Ciencias de la Comunicación. Evidentemente restaría hacer una crítica a la TGS, como es la ausencia en detenerse en aspectos comunicativos internos y definir de forma clara el papel del lenguaje, en el caso de un sistema social, del lenguaje simbólico. Otra ausencia, sería el ahondar en el hecho de que el sistema tenga 
internamente una jerarquía. La presencia de jerarquías permite la constitución de un todo relacionado con sus partes y con un conjunto de partes que operan bajo un sentido orientado al todo, no necesariamente en el centro, sino distribuido en el sistema.

\section{CONCLUSIONES}

Es muy importante en el trabajo científico tener presente que las ciencias estudian sistemas y relaciones de sistemas, no objetos aislados. Desde este punto de partida, hemos hecho una propuesta desde la que adentrarnos en la Teoría General de Sistemas (TGS), pasando luego después a resalar la diferencia entre sistema y entorno mediante una segunda diferencia constitutiva: la que existe entre elemento y relación.

Cualquier análisis teórico-sistémico debe consistir en la diferencia entre sistema y entorno, si bien en la actualidad se discreparía sobre una diferencia clara entre ambos. Los sistemas están estructuralmente orientados al entorno, que es donde cohabitan y sin él, no podrían existir, por lo tanto, no se trata de un contacto ocasional ni tampoco de una mera adaptación

Desde estas dos ideas, más las puntualizaciones concretas que se han hecho en este texto, esperamos que sirvan para suscitar en el autor, la necesidad de insertar una perspectiva sistémica, en el análisis de cualquier fenómeno social. La cuestión no es hablar de sistema social, sino concebir la realidad social así como su diversidad cultural, en una continua danza de sistema en conexión y reconstrucción constante, en el que nada es comprensible ni puede funcionar sin recurrir al todo y al entorno.

\section{BIBLIOGRAFÍA (APA)}

Luhmann, Niklas (1996). Introducción a la teoría de sistemas. Lecciones publicadas por Javier Torres Nafarrete .México: Anthropos/Universidad Iberoamericana.

Maturana, H (1975). "The Organization of the living: A theory of the living organization. The Int. J. of Man-Machine". Studies 7, pp. 313-332.

Maturana, H. y Varela, F. (1984). El árbol del conocimiento. Ed. Universitaria Santiago.

Melvin, L. De Fleur, Sandra J. Ball-Rokeach (1983). Teorías de la Comunicación de Masas. Paidós, Barcelona. 